\title{
The Method of Christopherson for Solving Free Boundary Problems for Infinite Journal Bearings by Means of Finite Differences
}

\author{
By Colin W. Cryer*
}

\begin{abstract}
A method for solving free boundary problems for journal bearings by means of finite differences has been proposed by Christopherson. We analyse Christopherson's method in detail for the case of an infinite journal bearing where the free boundary problem is as follows: Given $T>0$ and $h(t)$ find $\tau \in(0, T]$ and $p(t)$ such that (i) $\left[h^{3} p^{\prime}\right]^{\prime}=h^{\prime}$ for $t \in(0, \tau)$, (ii) $p(0)=0$, (iii) $p(t)=0$ for $t \in[\tau, T]$, and (iv) $p^{\prime}(\tau-0)=0$.

First, it is shown that the discrete approximation is accurate to $O\left([\Delta t]^{2}\right)$ where $\Delta t$ is the step size. Next, it is shown that the discrete problem is equivalent to a quadratic programming problem. Then, the iterative method for computing the discrete approximation is analysed. Finally, some numerical results are given.
\end{abstract}

1. Introduction. A journal bearing consists of a rotating cylinder which is separated from a "bearing surface" by a thin film of lubricating fluid (see Fig. 1). The fluid is fed in at $A$ and flows out at $B$. The width of the film is smallest at $C$, and we set $t=\theta / \theta_{C}$ where $\theta$ is as shown in Fig. 1 .

Between $C$ and $B$, the width of the film increases so that the pressure in the lubricating fluid may be expected to decrease. We assume that for $t=\tau$ the pressure becomes so low that the fluid vaporizes. The point $t=\tau$, the interface between the two phases of the fluid, is called the free boundary.

The mathematical problem can now be formulated (see Pinkus and Sternlicht [7, p. 41 and p. 46]): and

Problem 1. Find a function $p(t)$ and a constant $\tau$ such that $p \in \mathcal{e}[0, T] \cap \mathfrak{C}^{(2)}(0, \tau)$,

$$
\begin{aligned}
\mathscr{L} p(t) & =\frac{d}{d t}\left[h^{3}(t) \frac{d p}{d t}\right]-\frac{d h}{d t}=0, \quad 0<t<\tau, \\
p(t) & =0, \quad \tau \leqq t \leqq T, \\
p(0) & =0, \\
\frac{d}{d t} p(\tau) & =0 .
\end{aligned}
$$

In Problem 1, $p(t)$ is proportional to the fluid pressure, while Eq. (1.1) is Reynolds' equation for the pressure in a lubricating film.

Received February 13, 1970, revised January 19, 1971.

AMS 1970 subject classifications. Primary 65L10; Secondary 76D99, 76T05, 90C20.

Key words and phrases. Christopherson's method, free boundary problems, finite differences, journal bearings, quadratic programming, lubrication theory.

* Sponsored by the Mathematics Research Center, United States Army, Madison, Wisconsin, under Contract No. DA-31-124-ARO-D-462, and the Office of Naval Research, under Contract No. N00014-47-A-0128-0004.

Copyright @ 1971, American Mathematical Society 


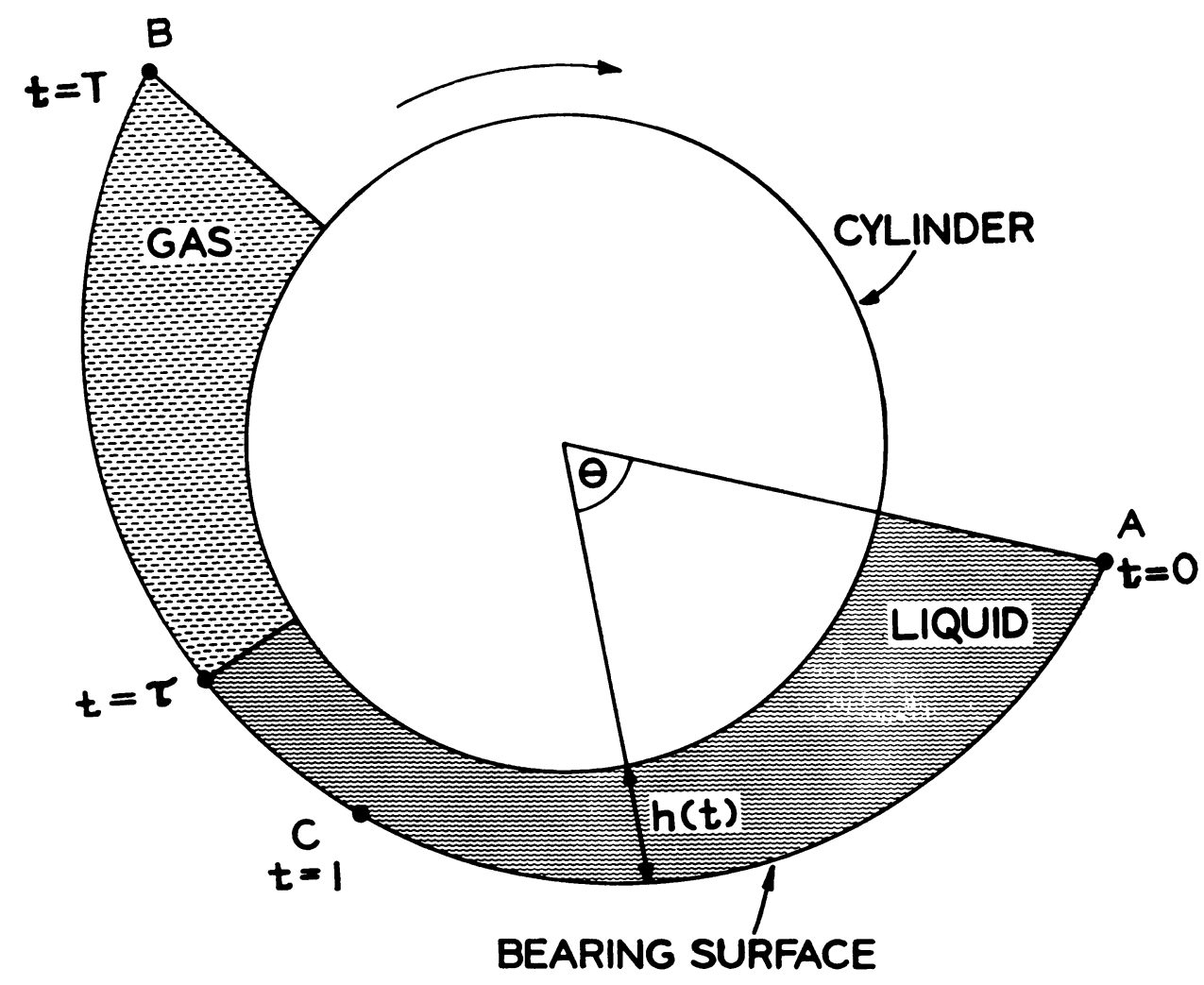

FIGURE 1. Cross-section of a journal bearing

In order that Problem 1 be well defined, it is necessary that $h(t)$, the width of the film, satisfy certain conditions. Throughout this paper, we will assume that $h \in \mathfrak{e}^{(1)}[0, T]$ and that

$$
\begin{array}{ll}
h(t)>0, & t \in[0, T], \\
\frac{d h}{d t}<0, & t \in(0,1), \\
\frac{d h}{d t}>0, & t \in(1, T), \\
h(T) \geqq h(0) . &
\end{array}
$$

It will be shown in Section 2 that conditions (1.5) through (1.7) ensure that there exists a unique solution to Problem 1.

Conditions (1.5) and (1.6) are always satisfied in practice, but this is not true of (1.7). However, as we shall see in Section 2, condition (1.7) can be imposed without any loss of generality.

In 1941, Christopherson [3] proposed a method for solving journal-bearing problems numerically. A partial analysis of the method was given by Gnanadoss and Osborne [6]. In the present paper, we present a detailed analysis of Christopherson's method as applied to Problem 1. 
This paper is based upon a technical report (Cryer [4]) in which further details, in particular all proofs, will be found.

Acknowledgement. The author is indebted to T. Ladner who wrote the program used to obtain the numerical results in Section 7.

2. The Analytic Problem. In this section, we first analyse Problem 1 and then formulate an equivalent problem, Problem 2.

Let

$$
\begin{array}{rlrl}
\vartheta_{2}(t) & =\int_{0}^{t} h^{-2}(s) d s, & & t \in[0, T], \\
\vartheta_{3}(t) & =\int_{0}^{t} h^{-3}(s) d s, & & t \in[0, T], \\
\varphi(t) & =\vartheta_{2}(t) / \vartheta_{3}(t), & & t \in(0, T], \\
\psi(t) & =h(t) \vartheta_{3}(t)-\vartheta_{2}(t), & t \in[0, T] .
\end{array}
$$

LEMMA 2.1. There is a unique constant $\sigma, 1<\sigma<T$, such that (i) $\psi(t)<0$, for $t \in(0, \sigma)$; (ii) $\psi(\sigma)=0$; and (iii) $\psi(t)>0$, for $t \in(\sigma, T]$.

For $a \in(0, T]$, let $q(t ; a)$ be the function such that (i) $\& q(t ; a)=0, t \in(0, a)$, (ii) $q(0 ; a)=0$; and (iii) $q(t ; a)=0, t \in[a, T]$; where $\mathscr{L}$ is as in (1.1). It is easily proved that $q(t ; a)$ is unique and is given by

$$
\begin{aligned}
q(t ; a) & =\vartheta_{2}(t)-\varphi(a) \vartheta_{3}(t), & & t \in[0, a], \\
& =0, & & t \in[a, T] .
\end{aligned}
$$

TheOREM 2.2. There is exactly one solution $\{p(t), \tau\}$ of Problem 1. If $\sigma$ is as in Lemma 2.1, then $\tau=\sigma$, and $p(t)=q(t ; \sigma)$.

Proof. The proof is a straightforward generalization of previous results (Birkhoff and Hays [1, p. 132], Pinkus and Sternlicht [7, p. 46], and Gnanadoss and Osborne [6]).

Problem 2. Find $\{p(t), \tau\}$ such that

$$
\begin{aligned}
\tau & =\sup \{a \in(0, T]: q(t ; a) \geqq 0 \text { for } t \in[0, T]\}, \\
p(t) & =q(t ; \tau) .
\end{aligned}
$$

That is, find the largest interval in which a nonnegative solution of Reynolds' equation exists.

Problem 2 was first suggested by Gnanadoss and Osborne [6], and the next theorem is a generalization of their results:

THEOREM 2.3. Problems 1 and 2 are equivalent.

We conclude this section with a discussion of condition (1.7). First, we note that it is the role of condition (1.7) to ensure that the lubricating fluid occurs in both the liquid and gaseous phases. If (1.7) is not satisfied, then it is possible, for example if $B$ is close to $C$ (see Fig. 1), for the fluid to occur only in the liquid phase.

Secondly, we note that there is no loss of generality in assuming (1.7). For suppose that $\tilde{h}(t)$ and $\tilde{T}$ are such that (i) $\tilde{h} \in \mathrm{e}^{(1)}[0, \tilde{T}]$; (ii) $\tilde{h}(t)>0, t \in[0, \tilde{T}]$; (iii) $d \tilde{h} / d t<0$, $t \in(0,1)$; and (iv) $d \tilde{h} / d t>0, t \in(1, \tilde{T})$; but that $\tilde{h}(\tilde{T})<\tilde{h}(0)$. Let $T>\tilde{T}$ and $h \in \mathrm{e}^{(1)}[0, T]$ be such that (1.5) through (1.7) are satisfied and $h(t)=h(t)$ for 
$t \in[0, \tilde{T}]$; clearly, $h$ and $T$ can be chosen in many ways. Let Problem 1 and Problem $\tilde{1}$ be the problems corresponding to $h$ and $\tilde{h}$, respectively. Clearly, if Problem $\tilde{1}$ has a solution $\{\tilde{p}(t), \tilde{\tau}\}$, then Problem 1 has a solution $\{p(t), \tau\}$ where $\tau=\tilde{\tau}, p(t)=\tilde{p}(t)$ for $t \in[0, \tilde{T}]$, and $p(t)=0$ for $t \in(\tilde{T}, T]$. Hence, to solve Problem $\tilde{1}$, we may first find the solution $\{p(t), \tau\}$ of Problem 1; by Theorem 2.2, this solution exists. If $\tau>\tilde{T}$, Problem $\tilde{1}$ has no solution. On the other hand, if $\tau \leqq \tilde{T}$, then Problem $\tilde{1}$ has the solution $\{\tilde{p}(t), \tilde{\tau}\}$ where $\tilde{\tau}=\tau$ and $\tilde{p}(t)=p(t)$ for $t \in[0, \tilde{T}]$.

3. The Discrete Approximation. Let $\{p(t), \tau\}$ be the solution of Problems 1 and 2. We subdivide the interval $[0, T]$ into $N$ subintervals, each of length $\Delta t$, so that $N=T / \Delta t$. We seek an integer $m$ and an $(N+1)$-vector $\mathbf{P}=\left\{P_{i}\right\}, j=0,1, \cdots, N$, such that $m \Delta t \approx \tau$ and $P_{i} \approx p(j \Delta t)$.

We approximate the Reynolds' equation (1.1) by the finite-difference equation

$$
(L \mathrm{P})_{i}=\frac{1}{(\Delta t)^{2}} \Delta\left(h_{i-1 / 2}^{3} \nabla P_{i}\right)-\frac{1}{\Delta t} \Delta h_{i-1 / 2}=0, \quad 0<i<N,
$$

where $\Delta$ and $\nabla$ denote the forward and backward difference operators respectively, $h_{i}=h(i \Delta t)$, and $h_{i-1 / 2}=h\left(\left[i-\frac{1}{2}\right] \Delta t\right)$.

In order to avoid certain trivial possibilities, we assume that $\Delta t \leqq \frac{2}{3}$, and that $N \geqq 3$. We also make an additional assumption about $h(t)$, namely, that

$$
h(T-(\Delta t) / 2) \geqq h((\Delta t) / 2) .
$$

Assumption (3.2) is trivially satisfied by, if necessary, slightly increasing $T$ and modifying the definition of $h(t)$ appropriately (see Section 2 where a similar device is used).

Let

$$
\begin{array}{rlrl}
I_{2}(i) & =\sum_{i=1}^{i}\left(h_{i-1 / 2}\right)^{-2}, & & 0 \leqq i \leqq N, \\
I_{3}(i) & =\sum_{i=1}^{i}\left(h_{i-1 / 2}\right)^{-3}, & & 0 \leqq i \leqq N, \\
\Phi_{i} & =I_{2}(i) / I_{3}(i), & 1 \leqq i \leqq N, \\
\Psi_{i} & =h_{i+1 / 2} I_{3}(i)-I_{2}(i), & 0 \leqq i \leqq N-1,
\end{array}
$$

with the convention that

$$
\sum_{i=1}^{0}\left(h_{i-1 / 2}\right)^{-2}=\sum_{i=1}^{0}\left(h_{i-1 / 2}\right)^{-3}=0 .
$$

Noting the analogy between Eqs. (1.1) and (3.1) and between Eqs. (2.1) through (2.4) and Eqs. (3.3) through (3.6), we are led to

LEMMA 3.1. There is a unique integer $n, 1-3(\Delta t) / 2 \leqq n \Delta t \leqq T-2(\Delta t)$, such that (i) $\Psi_{i}<0$, for $1 \leqq i<n$; (ii) $\Psi_{n} \leqq 0$; and (iii) $\Psi_{i}>0$, for $n<i \leqq N-1$.

For any integer $l, 1 \leqq l \leqq N$, let $\mathrm{Q}(l)=\left\{Q_{i}(l)\right\}$ be the $(N+1)$-vector such that

$$
\begin{aligned}
{[L Q(l)]_{i} } & =0, & 1 \leqq i \leqq l-1, \\
Q_{0}(l) & =0, & \\
Q_{i}(l) & =0, & l \leqq i \leqq N,
\end{aligned}
$$


where $L$ is as in (3.1). It is easily proved that $\mathrm{Q}(l)$ is unique and is given by

$$
\begin{aligned}
Q_{i}(l) & =\Delta t\left\{I_{2}(i)-\Phi_{l} I_{3}(i)\right\}, & & 0 \leqq i \leqq l, \\
& =0, & & l \leqq i \leqq N .
\end{aligned}
$$

We can now formulate the discrete analog of Problem 2:

Problem 2D. Find $\{\mathbf{P}, m\}$ such that,

$$
\begin{aligned}
& m=\max \left\{l: 1 \leqq l \leqq N ; Q_{i}(l) \geqq 0 \text { for } 0 \leqq i \leqq N\right\}, \\
& \mathbf{P}=\mathbf{Q}(m) .
\end{aligned}
$$

Theorem 3.2. There is exactly one solution $\{\mathbf{P}, m\}$ of Problem 2D. If $n$ is as in Lemma 3.1, then $m=n+1$, and $\mathrm{P}=\mathrm{Q}(m)$.

4. Error Analysis. In Theorems 2.2 and 3.2, explicit expressions for the solution $\{p(t), \tau\}$ of Problem 2 and the solution $\{\mathrm{P}, m\}$ of Problem 2D are given. By comparing these expressions, error estimates can be obtained. Under the assumption that

$$
h \in \mathfrak{e}^{(2)}[0, T]
$$

lengthy, but straightforward, computations yield

THEOREM 4.1. There are positive constants $(\Delta t)_{0}$ and $K$ such that if

$$
\Delta t \leqq(\Delta t)_{0},
$$

then

$$
|\tau-m \Delta t| \leqq 5(\Delta t) / 8
$$

and

$$
\left|p(j \Delta t)-P_{j}\right| \leqq K(\Delta t)^{2}, \quad 0 \leqq j \leqq N .
$$

It follows from Theorem 4.1 that $\{\mathrm{P}, m\}$ is as accurate an approximation to $\{p(t), \tau\}$ as could be hoped for. For we can at best have that

$$
|\tau-m \Delta t| \leqq(\Delta t) / 2,
$$

and (4.3) is almost as good as (4.5). Since we can at best have (4.5), we might expect that $\left|p(j \Delta t)-P_{j}\right|=O(\Delta t)$. Instead, the gods have smiled, and we have (4.4). More prosaically, the "reason" why (4.4) holds is that the condition $p^{\prime}(\tau)=0$ makes $p$ "relatively insensitive to errors in $\tau$. ."

5. Another Discrete Approximation. In this section, we formulate a second discrete approximation to Problem 2, Problem 3D, which can be shown to be equivalent to Problem 2D. The reason for introducing Problem 3D is that Christopherson's method is best understood if it is regarded as an algorithm for solving Problem 3D.

Set $M=N-1$ and denote by $\mathrm{A}$ the $M \times M$ matrix with components

$$
\begin{aligned}
\boldsymbol{A}_{i j} & =-\left(h_{i+1 / 2}\right)^{3}, & & \text { if } j=i+1, \\
& =\left[\left(h_{i+1 / 2}\right)^{3}+\left(h_{i-1 / 2}\right)^{3}\right], & & \text { if } j=i, \\
& =-\left(h_{i-1 / 2}\right)^{3}, & & \text { if } j=i-1, \\
& =0, & & \text { otherwise, }
\end{aligned}
$$


for $1 \leqq i, j \leqq M$. Noting (1.5), we see that $\mathbf{A}$ is a symmetric irreducibly diagonally dominant matrix with positive diagonal entries, so that $\mathbf{A}$ is positive definite (Varga $[9$, p. 23]).

Denote by B the $M$-vector with components

$$
B_{i}=-(\Delta t)\left[h_{i+1 / 2}-h_{i-1 / 2}\right], \quad 1 \leqq i \leqq M .
$$

Problem 3D. Find $M$-vectors $\mathrm{X}$ and $\mathrm{Y}$ such that

$$
\begin{aligned}
\mathbf{A X}-\mathbf{Y} & =\mathbf{B}, \\
\mathbf{X}^{T} \mathbf{Y} & =0, \\
\mathbf{X} & \geqq 0, \quad \mathbf{Y} \geqq 0 .
\end{aligned}
$$

Using the theory of quadratic programming and the detailed information available concerning the solution of Problem 2D, we obtain

THEOREM 5.1. Problem 3D has a unique solution. Problems 2D and 3D are equivalent. If $\{\mathrm{P}, m\}$ and $\{\mathrm{X}, \mathrm{Y}\}$ are the solutions of Problems $2 \mathrm{D}$ and $3 \mathrm{D}$, respectively, then

$$
\begin{aligned}
X_{i} & =P_{i}, \quad 1 \leqq i \leqq M, \\
\mathrm{Y} & =\mathrm{AX}-\mathrm{B}, \\
P_{0} & =P_{N}=0 \\
m & =\inf \left\{i ; \quad Y_{i}>0\right\} \\
Y_{i} & =0, \quad 1 \leqq i<m, \\
Y_{i} & >0, \quad m \leqq i \leqq M, \\
X_{i} & >0, \quad 1 \leqq i \leqq m-2, \\
X_{m-1} & =-(\Delta t) \Psi_{m-1} /\left\{\left(h_{m-1 / 2}\right)^{3} I_{3}(m)\right\} \geqq 0, \\
X_{i} & =0, \quad m \leqq i \leqq M .
\end{aligned}
$$

6. The Iterative Solution of the Discrete Approximation. In this section, we analyse the algorithm used by Christopherson to compute the solution of the discrete approximations to Problem 1.

Algorithm 6.1. Choose an $M$-vector $\mathrm{X}^{(0)}=\left\{X_{i}^{(0)}\right\}$ where $\mathrm{X}^{(0)} \geqq 0$. Choose a relaxation parameter $\omega$, where $0<\omega<2$.

Generate a sequence of $M$-vectors $\mathrm{X}^{(k)}=\left\{X_{i}^{(k)}\right\}, \mathbf{R}^{(k)}=\left\{R_{i}^{(k)}\right\}$, and $\mathrm{Y}^{(k)}=$ $\left\{Y_{i}^{(k)}\right\}, k=1,2, \cdots$, using the equations,

$$
\begin{aligned}
& R_{i}^{(k+1)}=B_{i}-\sum_{i=1}^{i-1} A_{i j} X_{i}^{(k+1)}-\sum_{i=1}^{M} A_{i j} X_{i}^{(k)}, \\
& X_{i}^{(k+1)}=\max \left\{0, X_{i}^{(k)}+\omega R_{i}^{(k+1)} / A_{i i}\right\}, \\
& Y_{i}^{(k+1)}=-R_{i}^{(k+1)}+A_{i i}\left(X_{i}^{(k+1)}-X_{i}^{(k)}\right) .
\end{aligned}
$$

The reader will have observed that Algorithm 6.1 consists of applying S.O.R. (systematic overrelaxation) to the equations $\mathbf{A X}=\mathbf{B}$ with the proviso that the iterates $\mathrm{X}^{(k)}$ be nonnegative. This was the way in which Algorithm 6.1 was viewed by 
Christopherson except that, since he worked by hand, he used relaxation rather than S.O.R. The condition that the vectors $\mathbf{X}^{(k)}$ be nonnegative arises naturally from the physical restraint that the lubricating fluid cannot support negative pressures.

Christopherson used Algorithm 6.1 without explicitly formulating the discrete problem that he was solving. Of the two formulations of the discrete problem that we have developed, Problems 2D and 3D, it seems to us that Problem 3D lies closest in spirit to Christopherson's ideas.

In computations for Cameron and Wood [2], Fox (working by hand) used Algorithm 6.1 with relaxation instead of S.O.R.; Raimondi and Boyd [8] (using an IBM 704) used the Liebmann or Gauss-Seidel method instead of S.O.R.; finally, the use of S.O.R. was suggested by Gnanadoss and Osborne [6].

Throughout the remainder of this section, we denote the solutions of Problems $2 \mathrm{D}$ and $3 \mathrm{D}$ by $\{\mathrm{P}, m\}$ and $\{\mathrm{X}, \mathrm{Y}\}$, respectively, and assume that $\mathrm{X}^{(k)}$ and $\mathrm{Y}^{(k)}$ are generated using Algorithm 6.1.

Using the results of Cryer [5], we obtain

THEOREM 6.1. For any $\mathrm{X}^{(0)} \geqq 0, \mathrm{X}^{(k)} \rightarrow \mathrm{X}$ and $\mathrm{Y}^{(k)} \rightarrow \mathrm{Y}$ as $k \rightarrow \infty$.

Next, we consider the speed with which Algorithm 6.1 converges. We define the asymptotic rate of convergence of Algorithm 6.1 to be

$$
R(\mathbf{A}, \mathbf{B}, \omega)=-\log \left\{\sup _{\mathbf{X}(0) \geq 0} \limsup _{k \rightarrow \infty}\left\|\mathbf{X}^{(k)}-\mathbf{X}\right\|^{1 / k}\right\},
$$

where $\|\cdot\|$ denotes any vector norm.

We need certain concepts from the theory of S.O.R. (see Varga [9]). Let $\tilde{\mathbf{A}}$ be a $p \times p$ positive definite matrix. Let $\tilde{\mathbf{A}}=\tilde{\mathbf{D}}-\tilde{\mathbf{E}}-\tilde{\mathbf{F}}$, where $\tilde{\mathbf{D}}$ is a diagonal matrix while $\tilde{\mathbf{E}}$ and $\tilde{\mathbf{F}}$ are, respectively, strictly upper and strictly lower triangular matrices. Then the point successive relaxation matrix corresponding to $\tilde{\mathbf{A}}$ is given by

$$
\mathfrak{L}_{\omega}(\tilde{\mathbf{A}})=(\tilde{\mathbf{D}}-\omega \tilde{\mathbf{E}})^{-1}\{(1-\omega) \tilde{\mathbf{D}}+\omega \tilde{\mathbf{F}}\} .
$$

The point Jacobi matrix $\tilde{\mathrm{J}}$ is given by

$$
\tilde{\mathrm{J}}=(\tilde{\mathrm{D}})^{-1}[\tilde{\mathrm{E}}+\tilde{\mathbf{F}}] \text {. }
$$

The asymptotic rate of convergence for $\mathscr{L}_{\omega}(\tilde{\mathrm{A}})$ is given by

$$
R_{\infty}\left[\mathscr{L}_{\omega}(\tilde{\mathrm{A}})\right]=-\log \left\{\rho\left[\mathcal{L}_{\omega}(\overline{\mathrm{A}})\right]\right\},
$$

where $\rho\left[\mathscr{L}_{\omega}(\tilde{\mathbf{A}})\right]$ is the spectral radius of $\mathscr{L}_{\omega}(\tilde{\mathbf{A}})$. Finally, the optimum relaxation parameter $\omega_{b}=\omega_{b}(\tilde{\mathrm{A}})$ satisfies

$$
R_{\infty}\left[\mathscr{L}_{\omega_{b}}(\tilde{\AA})\right]=\max _{\omega} R_{\infty}\left[\mathscr{L}_{\omega}(\tilde{\mathrm{A}})\right] .
$$

THEOREM 6.2. Assume that $\Psi_{m-1}>0$. Then

$$
R(\mathrm{~A}, \mathrm{~B}, \omega)=R_{\infty}\left(\mathscr{L}_{\omega}\left[\mathrm{A}^{(m-1)}\right]\right) \text {, }
$$

where $\mathrm{A}^{(m-1)}$ is the $(m-1) \times(m-1)$ matrix consisting of the first $(m-1)$ rows and columns of $\mathbf{A}$. If $\omega_{\text {opt }}=\omega_{b}\left(\mathrm{~A}^{(m-1)}\right)$, then $\omega_{\text {opt }} \leqq \omega_{b}(\mathbf{A})$ and $R(\mathbf{A}, \mathbf{B}, \omega) \leqq R\left(\mathbf{A}, \mathbf{B}, \omega_{\text {opt }}\right)$, $0<\omega<2$.

7. Numerical Results. In this section, we present numerical results for an infinitely long full journal bearing to illustrate the theoretical results of the preceding sections. 
The equations for an infinitely long full journal bearing are (Pinkus and Sternlicht [7, p. 42 and p. 46])

$$
\begin{array}{rlrl}
\frac{d}{d \theta}\left[w^{3}(\theta) \frac{d p}{d \theta}\right] & =\frac{d w(\theta)}{d \theta}, & & 0<\theta<\theta_{2}, \\
p(\theta) & =0, & & \theta_{2} \leqq \theta \leqq 2 \pi, \\
p(0) & =0, & & \\
\frac{d}{d \theta} p(\theta) & =0, & \theta=\theta_{2}, \\
w(\theta) & =1+\epsilon \cos \theta .
\end{array}
$$

Here, $\epsilon$ is the eccentricity ratio and satisfies $0 \leqq \epsilon<1$.

Introducing the variable

$$
t=\theta / \pi
$$

it is found that $p$ satisfies Eqs. (1.1) through (1.4) with $T=2, \tau=\theta_{2} / \pi$, and

$$
h(t)=(1+\epsilon \cos \pi t) / \sqrt{ } \pi .
$$

It is easily verified that $h$ satisfies (1.5), (1.6), (1.7), (3.2), and (4.1), so that all the results of the preceding sections are valid.

For $h$ given by (7.7), the solution of Problem 1 can be given analytically (Pinkus and Sternlicht [7, p. 47]); the expressions are complex and will not be given here.

Numerical results were obtained for the case $\epsilon=.8$. The computations were performed on the UNIVAC 1108 computer at the University of Wisconsin; this computer uses eight-decimal floating-point arithmetic. The analytic solution $\{p(t), \tau\}$ was computed directly. The discrete approximation $\{\mathbf{P}, m\}$ was computed by using Algorithm 6.1; the iterations were terminated when

$$
\left\|\mathbf{R}^{(k+1)}\right\|_{\infty}=\max _{i}\left|R_{i}^{(k+1)}\right| \leqq 10^{-7} .
$$

The initial approximation $\mathbf{X}^{(0)}$ was always taken to be identically zero.

Two experiments were carried out. In the first experiment, $N$ was taken equal to 64 while $\omega$ was varied. We were primarily interested in determining the number of iterations required to converge, that is the number of iterations required before (7.8) was satisfied. The results (see Table 7.1) showed that a reasonable strategy was to set $\omega=\omega^{*}$ where

$$
\omega^{*}=2 /\{1+\sin (\pi / N)\} .
$$

( $\omega^{*}$ is the optimum overrelaxation parameter for the discrete two-point boundaryvalue problem $\nabla \Delta u_{i}=b_{i}, 1 \leqq j \leqq N-1 ; u_{0}=u_{N}=0$.)

In the second experiment, $\omega$ was taken equal to $\omega^{*}$ while $N$ was varied. We were primarily interested in the difference between $\{p, \tau\}$ and $\{\mathbf{P}, m\}$. Setting

$$
\|p-\mathbf{P}\|_{\infty}=\max _{i}\left|p(j \Delta t)-P_{i}\right|,
$$

the dependence of $\|p-\mathrm{P}\|_{\infty}$ upon $\Delta t$ is shown in Table 7.2.

Bearing in mind that the UNIVAC 1108 works to only eight decimal places, it is clear that the results are in agreement with the assertion of Theorem 4.1 that 
TABLE 7.1. Number of iterations to converge $(N=64)$

\begin{tabular}{cc}
\hline$\omega$ & $\begin{array}{c}\text { No. of iterations to } \\
\text { converge }\end{array}$ \\
\hline 1.0 & 811 \\
1.1 & 670 \\
1.2 & 558 \\
1.3 & 453 \\
1.4 & 362 \\
1.5 & 288 \\
1.6 & 216 \\
1.7 & 146 \\
1.8 & 70 \\
1.9 & 136 \\
$\omega^{*}=1.90645$ & 146 \\
\hline
\end{tabular}

TABLE 7.2. Dependence of $\|p-\mathrm{P}\|_{\infty}$ upon $\Delta t$

\begin{tabular}{rlcc}
\hline \multicolumn{1}{c}{$N$} & \multicolumn{1}{c}{$\Delta t$} & $\|p-\mathrm{P}\|_{\infty}$ & $\begin{array}{c}\text { No. of iterations } \\
\text { to converge }\end{array}$ \\
\hline 64 & .03125 & .016017 & 146 \\
128 & .015625 & .002725 & 268 \\
256 & .0078125 & .000756 & 513 \\
512 & .00390625 & .000170 & 923 \\
1024 & .001953125 & .000073 & 1640 \\
\hline
\end{tabular}

$\|p-\mathrm{P}\|_{\infty} \leqq K(\Delta t)^{2}$. The other assertion of Theorem 4.1, namely that $|\tau-m \Delta t| \leqq$ $5(\Delta t) / 8$, was always satisfied.

Computer Sciences Department

The University of Wisconsin

Madison, Wisconsin 53706

1. G. BIRKHOFF \& D. F. HAYS, "Free boundaries in partial lubrication," J. Mathematical Phys., v. 42, 1963, pp. 126-138. MR 27 \#3168.

2. A. CAMERON \& W. L. Wood, "The full journal bearing," Inst. Mech. Engrs. J. Proc., v. 161,1949 , pp. 59-64.

3. D. G. Christopherson, "A new mathematical method for the solution of film lubrication problems," Inst. Mech. Engrs. J. Proc., v. 146, 1941, pp. 126-135. MR 3, 285.

4. C. W. CRYER, The Method of Christopherson for Solving Free Boundary Problems for Infinite Journal Bearings by Means of Finite Differences, Technical Report \#72, Computer Sciences Dept., University of Wisconsin, Madison, Wisconsin, 1969.

5. C. W. CRYER, "The solution of a quadratic programming problem using systematic overrelaxation," SIAM J. Control (To appear.)

6. A. A. GNANAdoss \& M. R. OsBORNE, "The numerical solution of Reynolds' equation for a journal bearing," Quart. J. Mech. Appl. Math., v. 17, 1964, pp. 241-246.

7. O. Pinkus \& B. StERnlicht, Theory of Hydrodynamic Lubrication, McGraw-Hill, New York, 1961.

8. A. A. RAImond \& J. Boyd, "A solution for the finite journal bearing and its application to analysis and design. III, Trans. Amer. Soc. Lubrication Engrs., v. 1, 1958, pp. 194-209.

9. R. S. VARgA, Matrix Iterative Analysis, Prentice-Hall, Englewood Cliffs, N. J., 1962. MR 28 \# 1725. 